\title{
Genetic reactivation of cone photoreceptors restores complex visual responses in Retinitis pigmentosa
}

Volker Busskamp ${ }^{1,2, \#}$, Jens Duebel ${ }^{1, \#}$, David Balya ${ }^{1, \#}$, Mathias Fradot ${ }^{3,4,5}$, Tim James

Viney $^{1}$, Sandra Siegert ${ }^{1}$, Anna C. Groner ${ }^{2,6}$, Erik Cabuy ${ }^{1}$, Valérie Forster ${ }^{3,4,5}$, Mathias

Seeliger $^{7}$, Martin Biel ${ }^{8}$, Peter Humphries ${ }^{9}$, Michel Paques ${ }^{3,4,5,10,11}$, Saddek Mohand-

Said $^{3,4,5,10}$, Didier Trono ${ }^{2,6}$, Karl Deisseroth ${ }^{12}$ José A. Sahel $^{3,4,5,10,11}$, Serge Picaud ${ }^{3,4,5,11}$

\& Botond Roska ${ }^{1}$

${ }^{1}$ Neural Circuit Laboratories, Friedrich Miescher Institute for Biomedical Research,

Basel, Switzerland

${ }^{2}$ NCCR Frontiers in Genetics Program

${ }^{3}$ Inserm, UMR_S968, Institut de la Vision, Paris, France

${ }^{4}$ UPMC Univ Paris 06, UMR_S 968, Institut de la Vision, Paris, F-75012, France

${ }^{5}$ CNRS, UMR_7210, Paris, F-75012, France

${ }^{6}$ School of Life Sciences, Ecole Polytechnique Fédérale de Lausanne, Lausanne,

Switzerland

${ }^{7}$ Diagnostics Research Group, Department of Ophthalmology II, Eberhard-Karls

University, Tübingen, Germany

${ }^{8}$ Center for Integrated Protein Science CIPS-M and Department of Pharmacy, Ludwig-

Maximilians-Universität München, Munich, Germany

${ }^{9}$ Smurfit Institute of Genetics, Trinity College, Dublin, Ireland

${ }^{10}$ Centre d'Investigation Clinique 503, Inserm-Centre Hospitalier National

d'Ophtalmologie des Quinze-Vingts, Paris, France 
${ }^{11}$ Fondation Ophtalmologique Adolphe de Rothschild, Paris, France

${ }^{12}$ Department of Bioengineering, Department of Psychiatry and Behavioral Sciences, Stanford University, Stanford, USA

${ }^{\text {\# }}$ These authors contributed equally to this work

Retinitis pigmentosa refers to a diverse group of hereditary diseases affecting two million people worldwide that lead to incurable blindness. As a common pathology, rod photoreceptors die early whereas light-insensitive, morphologically altered cone photoreceptors persist longer. It is unknown if these cones are accessible for therapeutic intervention. We show that expression of archaebacterial halorhodopsin in light-insensitive cones can substitute for the native phototransduction cascade and restore their light sensitivity in mouse models of Retinitis pigmentosa. Resensitized photoreceptors activate all retinal cone pathways, drive sophisticated retinal circuit functions including directional selectivity, activate cortical circuits, and mediate visually guided behaviors. Using human ex vivo retinas we show that halorhodopsin can reactivate light-insensitive human photoreceptors. Finally, we identified blind patients with persisting, lightinsensitive cones for potential halorhodopsin-based therapy.

Retinitis pigmentosa $(1,2)$ is the result of diverse mutations in more than 44 genes expressed in rod photoreceptors (3); these then degenerate, causing loss of night vision. Subsequently, cone photoreceptors, which are responsible for color and high acuity daytime vision, progressively lose their photoreceptive outer segments, leading to overall blindness. Despite this loss of sensitivity, cone cell bodies remain present longer 
than rods in both humans and animals (4-6), but it is not known whether these lightinsensitive cells can be reactivated or if information from them can still flow to downstream visual circuits (Fig. 1A) for a significant time window after the loss of photosensitivity (7).

To restore light-evoked activity in light-insensitive cone photoreceptors we genetically targeted a light-activated chloride pump (8-10), enhanced Natronomonas pharaonis halorhodopsin (eNpHR) $(11,12)$, to photoreceptors by means of adenoassociated viruses (AAVs) $(13,14)$. Light-activated chloride pumps are rational candidates for reactivating vertebrate photoreceptors as both eNpHR-expressing cells (12) and healthy photoreceptors hyperpolarize in response to increases in light intensity. We selected two animal models of Retinitis pigmentosa for gene therapy, both of which lead to retinal degeneration (RD). $\mathrm{Cnga3}^{-/-} ; \mathrm{Rho}^{-/-}$double-knockout mice served as a model of slow forms of RD (s-RD mice) (15), and $P d e 6 b^{\text {rd1 }}$ (also known as rd1) mice as a model of fast forms of RD (f-RD mice) (16). Targeted expression of eNpHR was accomplished using three cell-specific promoters (Fig. 1B): Human rhodopsin (hRHO) (17); human red opsin (hRO) (18); and mouse cone arrestin-3 (mCAR) (19).

To assess the effectiveness and specificity of the promoters, we used eNpHR fused to enhanced yellow fluorescent protein (11) (EYFP, Fig. 2A-I). It is important to restrict the expression of eNpHR to photoreceptors only since eNpHR in downstream retinal circuit elements, such as ON-bipolar and ON-ganglion cells, may inhibit the flow of information across the retina. Enhanced green fluorescent protein (EGFP)-expressing AAVs were used as controls throughout this study (Fig. S1). We selected the hRO and mCAR promoters for s-RD mice (Fig. 2B-C), and the mCAR promoter for f-RD mice (Fig. 2F), based on their ability to selectively drive expression of eNpHR-EYFP in a high percentage of cone photoreceptors (Fig. S2-S3). 
The lifespan of cones in RD mice defines the window of opportunity for reactivation. We therefore tested EYFP or EGFP expression driven by the mCAR promoter at different time points during retinal degeneration. Surprisingly, EYFP and EGFP expression lasted more than eight months (Fig. 3A, S4, last tested f-RD at P264, and s-RD at P255), longer than opsin protein expression ( $\sim \mathrm{P} 95$ (5) or between P21 and P110 (20), and data not shown). We isolated these long-lasting AAV-transduced cells from $\mathrm{f}-\mathrm{RD}$ retinas at different time points and verified their identity by analyzing their transcriptome (P110-P220, Fig. S5). Cone-specific genes were expressed in the isolated cells while markers of other retinal cell types were absent, suggesting that AAV-labeled cells are altered cones, even at later stages of degeneration. The fact that opsin mRNA remained, while the opsin protein didn't suggests translational down-regulation of opsins. We estimate that $\sim 27 \%$ of cones remain between P184 and P255 in s-RD, and $\sim 25 \%$ between P182 and P264 in f-RD mice (Fig. 3A).

We tested whether eNpHR drives cone light responses in RD retinas at an age (P53-P264, Table S1) when many (s-RD) (15) or all (f-RD) (20) rods have already died. eNpHR-EYFP-expressing photoreceptors in s-RD and f-RD retinas displayed large (Fig. 3B, S6), sustained (Fig. 3C), and significantly faster (Fig. 3D) photocurrents than wildtype cones. The photocurrents peaked at $580 \mathrm{~nm}$. Photoreceptors expressing only EGFP did not react to light. The magnitude of photocurrents in eNpHR-transduced s-RD and fRD mice were similar (Fig. 3B). The current size was independent of the holding voltage (Fig. 3E), a finding that is consistent with the view that the photocurrents are mediated by ion pumps (10). Photocurrents and voltages were modulated across three logarithmic units of intensities (Fig. S6). In the absence of functional outer segments, which normally generate currents that depolarize photoreceptors in the dark, RD photoreceptors were expected to stay hyperpolarized. A hyperpolarized state would limit the ability of eNpHR currents to modulate synaptic transmission. However, the 
recorded eNpHR-expressing $\mathrm{RD}$ photoreceptors were depolarized in the dark $(-26 \pm 3$ $\mathrm{mV}, \mathrm{n}=12$, see Supplementary Discussion).

The ability of eNpHR-reactivated RD photoreceptors to convey information to downstream retinal circuits depends on the presence of functional photoreceptor-tobipolar cell synapses. As retinal degeneration progresses, these synapses morphologically reorganize (3). However, they still possess elements of both pre- and post-synaptic machinery (5). To test for potential signal flow from photoreceptors to ganglion cells, we recorded excitatory currents from ganglion cells, the output neurons of the retina. Ganglion cells in eNpHR-transduced s-RD and f-RD retinas displayed robust, light-evoked excitatory currents (Fig. 4A), indicating functional outer and inner retinal synaptic connections. The magnitudes of these excitatory currents were comparable to those in wild-type retinas (Fig. 4A). There were no measurable lightevoked currents in the ganglion cells of retinas transfected with EGFP (Fig. 4A).

The retina incorporates two major information channels that diverge at the level of bipolar cells, and are physically separated by depth within the inner plexiform layer (IPL). ON cells are activated by light increments, while OFF cells are activated by light decrements (21). The activity of ganglion cells is coded by action potentials ("spikes") that form the output signal of the retina (22) and the input signal to higher brain centers. Strikingly, light stimulation evoked excitatory currents and spiking activity in ganglion cells of eNpHR-transduced RD retinas either at light increments, light decrements, or both (P53-P264, Fig. 4A, B). The dendritic processes of morphologically reconstructed ganglion cells were properly aligned in the corresponding ON or OFF strata (23) of the IPL (Fig. 4B). Ganglion cell spike frequencies spanned similar ranges in s-RD, f-RD, and wild-type retinas (Fig. 4B). Some ganglion cell types respond preferentially to changes in illumination and therefore spike transiently after a light step while others represent the level of illumination and therefore spike in a more sustained manner. The 
dynamics of spiking activity in eNpHR-transduced retinas varied from transient to sustained, as in wild-type retinas (Fig. S7). Light-stimulation of eNpHR-reactivated photoreceptors in RD retinas even evoked ganglion cell spiking activity at later stages of degeneration (s-RD: $<$ P255, f-RD: $<$ P264, Fig. S7).

We next asked if basic forms of spatial processing were functional in the e-NpHRtransduced RD retinas. Lateral inhibition is a conserved feature of vertebrate retinas that is important for spatial contrast ("edge") enhancement. When spots of increasing sizes were presented to eNpHR-expressing RD retinas, the response magnitude of ganglion cells reached a maximum and then gradually decreased, a sign of lateral inhibition (21, 24) (Fig. 4C, S7). Lateral inhibition also results in ON-center OFF-surround responses (21) that we were also able to observe in eNpHR-expressing RD retinas (Fig. S7). Another example of spatial processing is the directional-selective responses of types of ganglion cells to motion stimuli (25). The activity of directional selective ganglion cells is important for the optokinetic reflex (26). When eNpHR-transduced RD retinas were stimulated with bars moving in different directions some ganglion cells responded preferentially to motion in a particular direction but produced little activity when the bar moved in the opposite direction (27) (Fig. 4D). This response asymmetry suggests that the retinal circuit for directional selectivity is, at least partially, maintained in $\mathrm{RD}$ retinas.

The sensitivity of eNpHR to light is less than that of normal cones (Fig. 4E), and while normal cones can adapt to different light intensities, eNpHR-driven cones have a fixed sensitivity range (see Supplementary Discussion). However, the sensitivity of ganglion cells to light was 1.7 log units higher than that of the eNpHR-expressing photoreceptors (Fig. 4E), and the light levels required for eNpHR stimulation at $580 \mathrm{~nm}$ are below the limit allowed for safe radiation of the human eye according to the "2006 European directives on artificial optical radiation" (Fig. 4E) (28). 
The slow and fast RD mouse models not only differ in the time course of photoreceptor degeneration, but also in the amount of light-driven activity present during development. s-RD mice have no light-sensitive rod-cone system during development (15) while f-RD mice lose the rod-cone function gradually, and are blind by four weeks of age (16). In the retina, the responses in eNpHR-activated s-RD and $\mathrm{f}$ $\mathrm{RD}$ mice were similar, suggesting that the development of the tested retinal functions, like direction selectivity, may not require light-driven input from rods and cones. Light stimulation of the eyes resulted in visually evoked potentials in eNpHR-expressing fRD mice but not in EGFP-transduced controls (P42-P118, Fig. S8). In contrast to the retina, we could not measure light-driven cortical activity in eNpHR-transduced s-RD mice.

We next evaluated whether light could induce behavioral changes in eNpHRtransduced mice. In dark-light box tests (29), eNpHR-expressing f-RD and s-RD mice performed significantly better than the corresponding EGFP-expressing control groups (P44-P143, Fig. 5A), and the increased performance depended on the illumination level (Fig. 5B). In the optomotor reflex test (30), only eNpHR-expressing f-RD mice performed better than EGFP-expressing control mice at a variety of drum speeds (P69P153, Fig. 5C). Wild-type (Fig. S8) and f-RD responses both peaked at the same speed. The optimum spatial frequency was higher for wild-type animals $(0.26 \mathrm{cpd})$ compared to $\mathrm{f}-\mathrm{RD}(0.13 \mathrm{cpd})$. These experiments demonstrated that resensitized photoreceptors are able to drive visually-guided behavior in $\mathrm{f}-\mathrm{RD}$ and, to some extent, in s-RD mice.

To test for potential toxicity of eNpHR or the unmodified NpHR, we first compared the retinas of eNpHR-transduced wild-type mice (six weeks after AAV administration) with those of normal wild-type mice. The number of photoreceptors was similar in both conditions (Fig. S9) and, in addition to the light-induced spiking activity, ganglion cells in eNpHR-transduced wild-type retinas had a wider action spectrum, a 
gain of function at longer wavelengths, than ganglion cells in uninjected wild-type retinas (Fig. S9). This suggests that both intrinsic opsins and eNpHR are at work. Next, we compared the retinas of transgenic mice expressing NpHR in photoreceptors under the control of a bovine rhodopsin promoter with wild-type retinas and found similar numbers of photoreceptors (at P140, Fig. S9). These results suggest that in the studied time window neither the unmodified NpHR nor eNpHR induce additional photoreceptor degeneration.

The translation of gene therapy achieved in mice to human subjects requires the use of promoters and AAV serotypes that drive photoreceptor-specific eNpHR expression in human retinas. We therefore tested our AAVs on human ex vivo retinal explants (Fig. 6A), which we could keep in culture for 2-3 weeks. Due to this short time window and the relatively long period of time required to efficiently express eNpHR from AAVs, we had to use immunohistochemistry to visualize eNpHR-EYFP protein expression in the cultured human retinas. Of the three promoters, mCAR directed expression of eNpHR specifically in human photoreceptors (Fig. S10). To reduce the time required to obtain robust eNpHR-EYFP expression, necessary for two-photon laser targeted electrophysiology, we inserted the mCAR-eNpHR construct into a lentiviral vector (31) (Fig. S10). Using this new vector, high levels of eNpHR expression were found specifically in human photoreceptors after only 1-2 days of incubation (Fig. 6B, S10). Brightly labeled photoreceptors in the parafoveal region displayed photocurrents and photovoltages with spectral tuning reflecting eNpHR activation (Fig. 6C, S10). We could not measure any photocurrents from control human retinas, even at the time when the retina was isolated.

To find potential patients with Retinitis pigmentosa eligible for eNpHR-mediated restoration of visual functions, we screened a database (see Supplementary Discussion) which contained records of retinal images acquired by optical coherence tomography 
(OCT), Goldman visual field tests, multifocal electroretinograms (ERG), full field ERG, and visual acuity tests. We identified legally blind patients (data from one of whom are shown in Fig. 6 and Fig. S11) with no visible outer segments on OCT pictures, but cone cell bodies in the central region. These criteria may be used in the future for selecting patients who could benefit from this therapy.

In summary, we have shown that a microbial gene introduced to surviving cone cell bodies reactivated retinal ON and OFF pathways and the retinal circuitry for lateral inhibition and directional selective responses. Moreover, the reactivated cones enabled $\mathrm{RD}$ mice to perform visually-guided behaviors. The tested time window of intervention was up to $\sim 260$ days in $\mathrm{f}-\mathrm{RD}$ and s-RD mice, suggesting that persisting cone cell bodies $(\sim 25 \%)$ are enough to induce ganglion cell activity, even during later stages of degeneration. Our finding that AAVs with the mCAR promoter specifically transduced human photoreceptors, and the identification of patients with little measurable visual function and no outer segments but surviving cone cell bodies, suggest a potential for translating eNpHR-based rescue of visual function to humans (see Supplementary Discussion). In the future, eNpHR-based restoration may be combined with other approaches that increase the survival of altered photoreceptors $(20,32-34)$.

Acknowledgements. We thank Brigitte Gross Scherf, Sabrina Djaffer, and Hubertus Kohler for technical assistance, and Pamela Lagali, Karl Farrow, Andrew Matus, and Sara Oakeley for their comments on the manuscript. pAAV2.1-Rho-EGFP was kindly provided by Alberto Auricchio. Jeremy Nathans kindly provided the pRed2.1lacZ. Post-mortem human retinas were kindly provided by Teja Wesseling and Elisabeth Pels at the Cornea Bank in Amsterdam (Netherlands). The study was supported by Friedrich Miescher Institute funds, a US Office of Naval Research Naval International Cooperative Opportunities in Science and Technology Program grant, a Marie Curie Excellence Grant, and an EU HEALTH-F2223156 Grant to B.R., a grant from the European Union (RETICIRC) to B.R. and S.P., grants from the Agence National de la recherché (MEDINAS, RETINE) to S.P., a Center Grant from Foundation Fighting 
Blindness (USA) to S.M-S. and J.A.S., grants from the Swiss National Science Foundation and the European Union to D.T., a Marie Curie Postdoctoral Fellowship to D.B., a National Centers of Competence in Research Frontiers in Genetics fellowship to V.B and A.C.G.

Correspondence and requests for materials should be addressed to B.R. (botond.roska@fmi.ch).

\section{Figure Legends}

Figure 1 Scheme of the excitatory pathways of cone retinal circuitry in wild-type and $\mathrm{RD}$ retinas. (A) Cone photoreceptors (cPR) in wild type retinas (left) detect light with photopigments in their outer segments (OS). Cone cell bodies are located in the outer nuclear layer (ONL). Cones provide input to ON and OFF bipolar cells (BC) that have cell bodies in the inner nuclear layer (INL). Bipolar cells are connected to corresponding ON and OFF ganglion cells (GC). Ganglion cell bodies are in the ganglion cell layer (GCL), and their axons relay visual information to higher visual centers. The locations of inhibitory interactions mediated by horizontal cells in the outer plexiform layer (OPL), and by amacrine cells in the inner plexiform layer (IPL) are indicated by gray boxes. Light-insensitive RD cones (right) lack outer segments but their cell bodies (green) persist longer than rods. (B) AAV-expression constructs. $\mathrm{hRHO}, \mathrm{hRO}$, or mCAR promoters drive eNpHR-EYFP.

Figure 2. Targeted expression of a light-sensitive chloride pump in persisting photoreceptors in RD retinas. (A-I) Cross-sections of GFP-immunostained (see Materials and methods) retinas transduced by hRHO- (A, D, G), hRO- (B, E, H) and mCAR- (C, F, I) eNpHR-EYFP AAVs in s-RD (A-C), f-RD (D-F), and wild-type (G-I) mice. Left panel of each pair, eNpHR-EYFP, right panel of each pair, co-stained with DAPI. Scale bar $20 \mu \mathrm{m}$. 
Figure 3. Light responses in surviving eNpHR-expressing RD cones. (A) Left, confocal top views of GFP-immunostained RD photoreceptors transduced by mCAR-eNpHREYFP in P255 s-RD (top) and P264 f-RD (bottom) retinas. Left panel of each pair, eNpHR-EYFP, right panel of each pair, co-stained with DAPI. Scale bar $20 \mu \mathrm{m}$. Right, estimated fraction of surviving cones (see Materials and methods) in s-RD and f-RD mice at different time points compared to wild-type cone numbers. " $n$ " refers to the number of retinas analyzed. (B) Left: photocurrent action spectrum of an eNpHR(black) or EGFP- (gray) expressing s-RD cone. Top, color-ramp; bottom, light flashes. Gray bars indicate the timing of the full-field light stimuli. Right: magnitude of photocurrents in photoreceptors expressing eNpHR or EGFP. Dashed lines show the peak magnitude of photocurrents in wild-type (wt) cones (short dashes) and wild-type rods (long dashes). (C) Peak and steady-state photocurrents ( $0.5 \mathrm{~s}$ flash) in RD and wild-type animals. (D) Rise and decay time constants of eNpHR-mediated photocurrents compared to the rise time constant in wild-type photoreceptors. (E) eNpHR-mediated photocurrents in RD retinas at different holding voltages. Since the response properties in s-RD and $\mathrm{f}-\mathrm{RD}$ mice were similar, the data from both mouse lines were grouped in panels (C-E). All wild-type cone response data shown in Fig. 3 were taken from (35).

Figure 4. Light responses in eNpHR-expressing RD retinas. For each functional test, individual examples are shown on the left followed by statistics on the right. (A) Ganglion cell excitatory currents. Left: light-evoked excitatory currents in ON (top), ON-OFF (middle) and OFF (bottom) ganglion cells in eNpHR-expressing s-RD retinas. Gray bars show the timing of the full-field light stimulus. Right: magnitude of lightevoked peak excitatory currents in s-RD, f-RD, and wild-type (wt) mice; ON, OFF, and ON-OFF cells are pooled together. Full-field stimulus. (B) Ganglion cell spiking output. Left: top, spike recordings (four repetitions) from ON (left) and OFF (right) ganglion cells from an eNpHR-expressing f-RD retina; middle, spike frequency responses (gray 
bar shows the timing of a full-field light stimulus); bottom, corresponding cells filled with neurobiotin (green), choline acetyltransferase (ChAT, magenta) as an IPL stratification marker, and DAPI-staining (white) to visualize nuclear layers. Right: peak spike frequency distribution in response to full-field flash stimuli in s-RD, $f-R D$, and wild-type retinas. (C) Lateral inhibition. Left: top, spike trains from an OFF ganglion cell in response to light stimulation with a white spot of increasing size (three repetitions); bottom, corresponding spike frequency. Right: number of spikes evoked by $1 \mathrm{~mm}$ diameter spot stimuli (center + surround stimulus) relative to the number of spikes evoked by a 0.1-0.2 mm spot (center stimulus). (D) Directional selective responses. Left: spike recordings during stimulation with a fast-moving bar (width is $200 \mu \mathrm{m}$, speed is $1.5 \mathrm{~mm} \mathrm{~s}^{-1}$, four repetitions) in the preferred direction (top) and in the opposite direction ("null" direction, bottom). Stimulus timing is shown by gray bars. Right: direction-selectivity index (see Materials and methods) (36). (E) Light sensitivity. eNpHR-induced excitatory current responses in ganglion cells and photocurrents in photoreceptors as a function of light intensity. The gray lines at the bottom display the ranges of sensitivities for rods (only partially shown), cones, and intrinsically photosensitive ganglion cells (ipRGCs). The maximum light intensity at $580 \mathrm{~nm}$ allowed in the human eye according to the "2006 European directives on artificial optical radiation" (28) is shown by the vertical dashed line. In all panels in Fig. 4 , " $n$ " refers to the number of different cells we measured from. Light intensity was $10^{16}$ photons $\mathrm{cm}^{-2} \mathrm{~s}^{-1}$ for each experiment.

Figure 5. Visually guided behavior in eNpHR-expressing RD mice. (A) Dark-light box experiment. Percentage of time the AAV-injected RD animals and wild-type (wt) mice spent in the light compartment. (B) Dark-light box test. Percentage of time mice spent in the light compartment as a function of light intensity. (C) Optomotor response score of eNpHR- or EGFP-expressing RD animals at different rotational speeds and spatial frequencies. In all panels of Fig. 5, " $n$ " refers to the number of different animals. 
Figure 6. Translational aspects of eNpHR-EYFP-mediated reactivation of photoreceptors. (A) Retinal slice from a human retinal explant ( $24 \mathrm{~h}$ post-mortem). Scale bar $30 \mu \mathrm{m}$. (B) Fluorescent live image of a lentivirus-transfected area from a human retina after 7 days in culture, and 2 days after lentiviral administration. Scale bar $20 \mu \mathrm{m}$.

(C) Action spectrum of an eNpHR-expressing human photoreceptor stimulated with full-field light flashes ranging from 450 to $650 \mathrm{~nm}$ (top: current response, bottom: voltage response). Gray bars indicate the timing of the stimulus. (D) Representative OCT scan covering the foveal region of a healthy individual. (E) Magnified image: IS, inner segments; OS, outer segments; RPE, retinal pigment epithelium. Scale bar 200 $\mu \mathrm{m}$. (F) OCT from the left eye of a 40-year old male patient with sporadic RP (loss of vision since the age of 15). Outer segments are undetectable.

\section{References}

1. K. Shintani, D. L. Shechtman, A. S. Gurwood, Optometry 80, 384 (Jul, 2009).

2. T. Cronin, T. Leveillard, J. A. Sahel, Curr Gene Ther 7, 121 (Apr, 2007).

3. R. E. Marc, B. W. Jones, C. B. Watt, E. Strettoi, Prog Retin Eye Res 22, 607 (Sep, 2003).

4. Z. Y. Li, I. J. Kljavin, A. H. Milam, J Neurosci 15, 5429 (Aug, 1995).

5. B. Lin, R. H. Masland, E. Strettoi, Exp Eye Res 88, 589 (Mar, 2009).

6. A. H. Milam, Z. Y. Li, R. N. Fariss, Prog Retin Eye Res 17, 175 (Apr, 1998).

7. $\quad$ E. Banin et al., Neuron 23, 549 (Jul, 1999).

8. E. Bamberg, J. Tittor, D. Oesterhelt, Proc Natl Acad Sci U S A 90, 639 (Jan 15, 1993).

9. B. Schobert, J. K. Lanyi, J Biol Chem 257, 10306 (Sep 10, 1982).

10. A. Seki et al., Biophys J 92, 2559 (Apr 1, 2007).

11. V. Gradinaru, K. R. Thompson, K. Deisseroth, Brain Cell Biol 36, 129 (Aug, 2008).

12. F. Zhang et al., Nature 446, 633 (Apr 5, 2007).

13. G. P. Gao et al., Proc Natl Acad Sci U S A 99, 11854 (Sep 3, 2002).

14. C. Lebherz, A. Maguire, W. Tang, J. Bennett, J. M. Wilson, J Gene Med 10, 375 (Apr, 2008).

15. E. Claes et al., Invest Ophthalmol Vis Sci 45, 2039 (Jun, 2004). 
16. D. B. Farber, J. G. Flannery, C. Bowes-Rickman, Prog Retin Eye Res 13, 31 (1994).

17. M. Allocca et al., J Virol 81, 11372 (Oct, 2007).

18. Y. Wang et al., Neuron 9, 429 (Sep, 1992).

19. X. Zhu et al., FEBS Lett 524, 116 (Jul 31, 2002).

20. C. Punzo, K. Kornacker, C. L. Cepko, Nat Neurosci 12, 44 (Jan, 2009).

21. S. W. Kuffler, J Neurophysiol 16, 37 (Jan, 1953).

22. T. Gollisch, M. Meister, Neuron 65, 150 (Jan 28).

23. E. V. Famiglietti, Jr., H. Kolb, Science 194, 193 (Oct 8, 1976).

24. H. B. Barlow, J Physiol 119, 69 (Jan, 1953).

25. J. B. Demb, Neuron 55, 179 (Jul 19, 2007).

26. K. Yoshida et al., Neuron 30, 771 (Jun, 2001).

27. H. B. Barlow, R. M. Hill, Science 139, 412 (Feb 1, 1963).

28. P. Degenaar et al., J Neural Eng 6, 035007 (Jun, 2009).

29. M. Bourin, M. Hascoet, Eur J Pharmacol 463, 55 (Feb 28, 2003).

30. J. Abdeljalil et al., Vision Res 45, 1439 (May, 2005).

31. R. Zufferey, D. Nagy, R. J. Mandel, L. Naldini, D. Trono, Nat Biotechnol 15, 871 (Sep, 1997).

32. B. Chen, C. L. Cepko, Science 323, 256 (Jan 9, 2009).

33. T. Leveillard, J. A. Sahel, Sci Transl Med 2, 26ps16 (Apr 7).

34. Y. Yang et al., Mol Ther 17, 787 (May, 2009).

35. S. S. Nikonov, R. Kholodenko, J. Lem, E. N. Pugh, Jr., J Gen Physiol 127, 359 (Apr, 2006).

36. W. R. Taylor, D. I. Vaney, J Neurosci 22, 7712 (Sep 1, 2002).

\section{Supporting Online Material}

www.sciencemag.org

Supplementary Discussion

Materials and methods

Figs. S1 - S1 1

Tables S1 - S4 
Figure 1

A

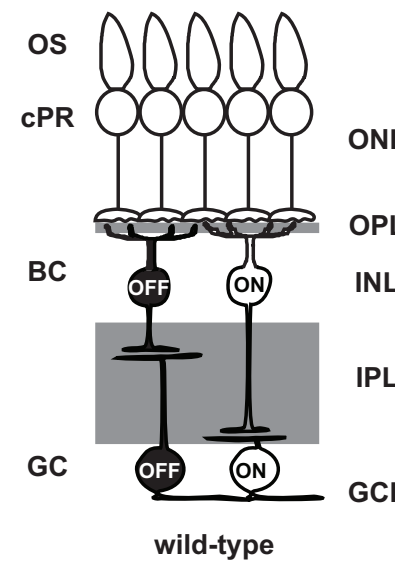

B

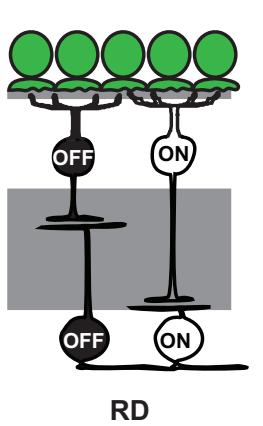

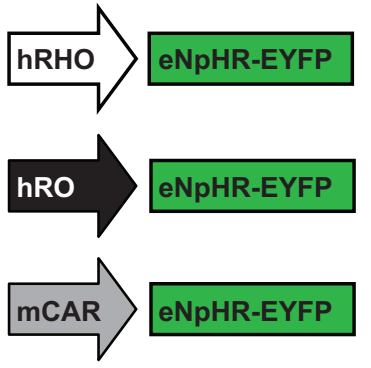


Figure 2
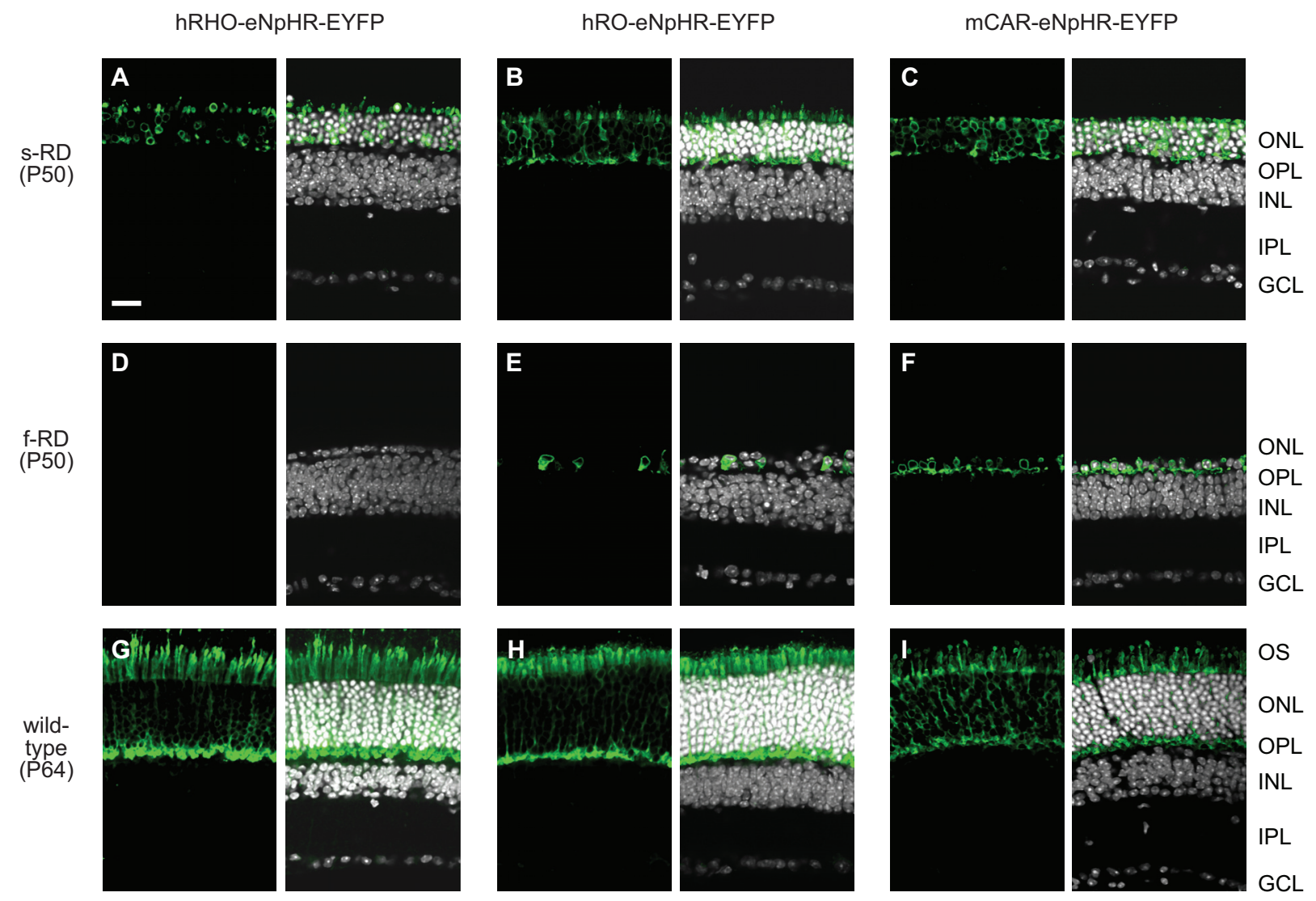
Figure 3

A
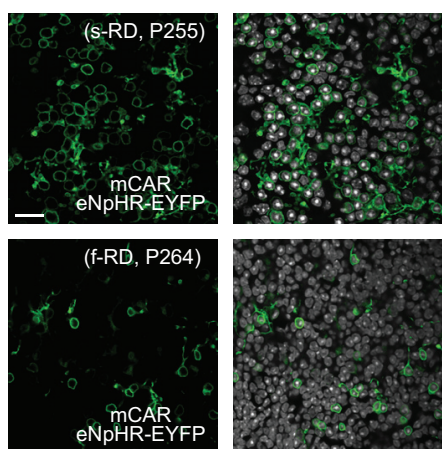

C

S-RD and f-RD wt

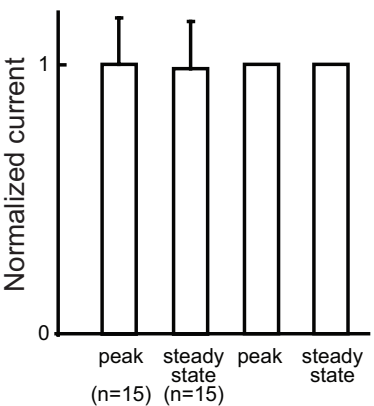

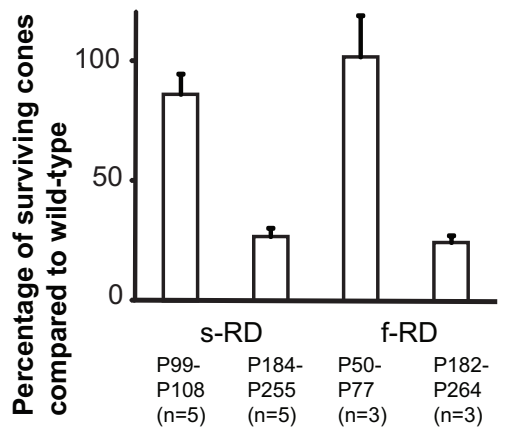

s-RD and f-RD
eNpHR

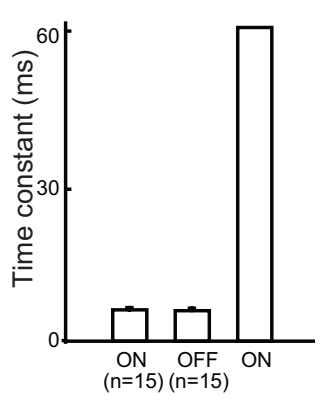

E $s-R D$ and f-RD

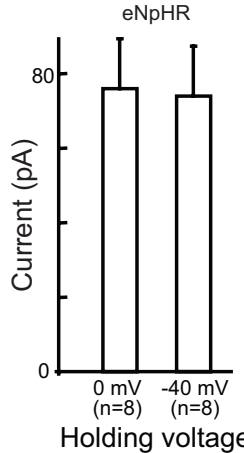

\section{B}
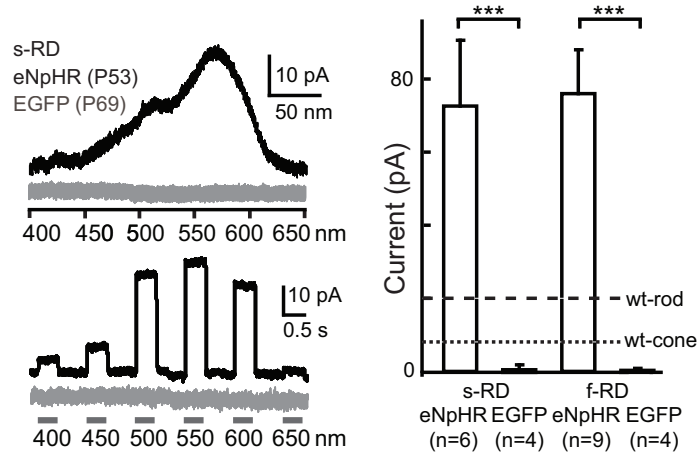


\section{Figure 4}

A

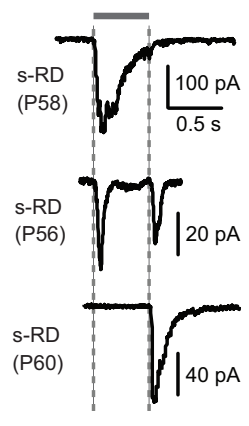

C
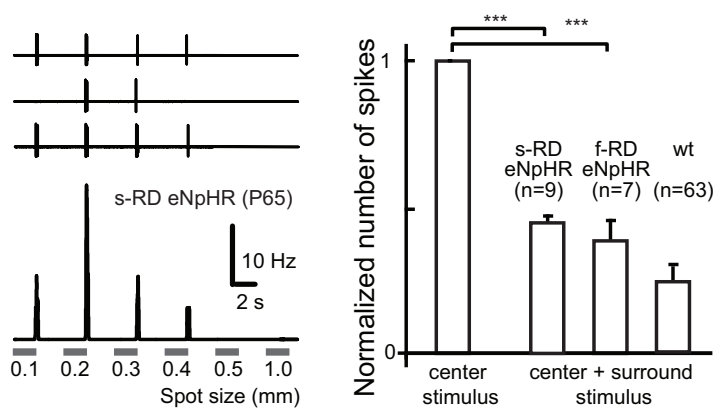

B

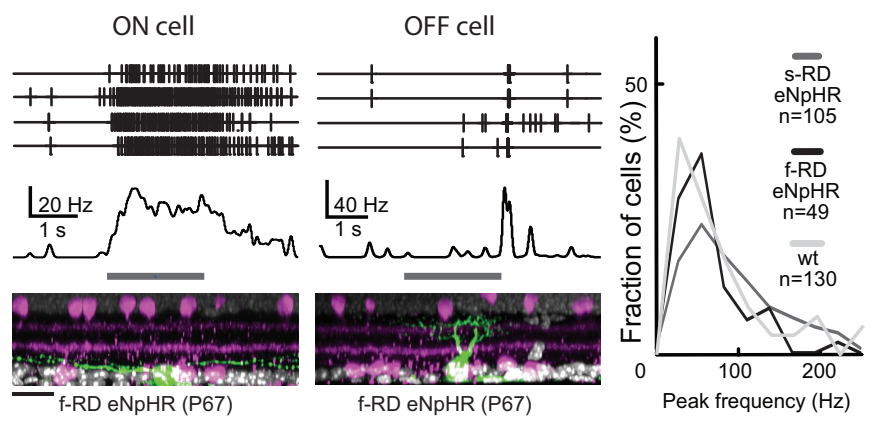

D

Preferred direction

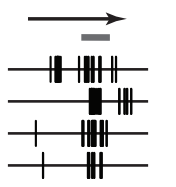

Null direction

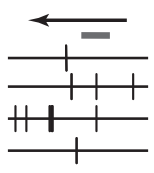

s-RD eNpHR (P122)

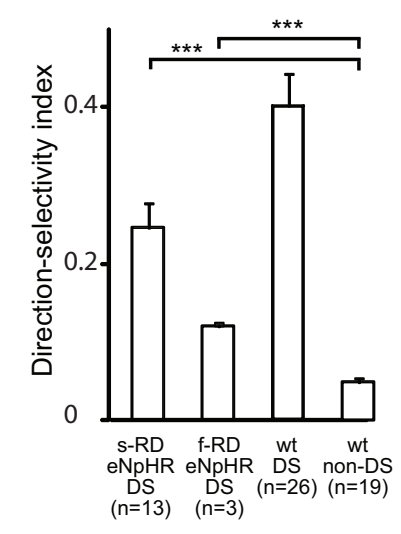

E

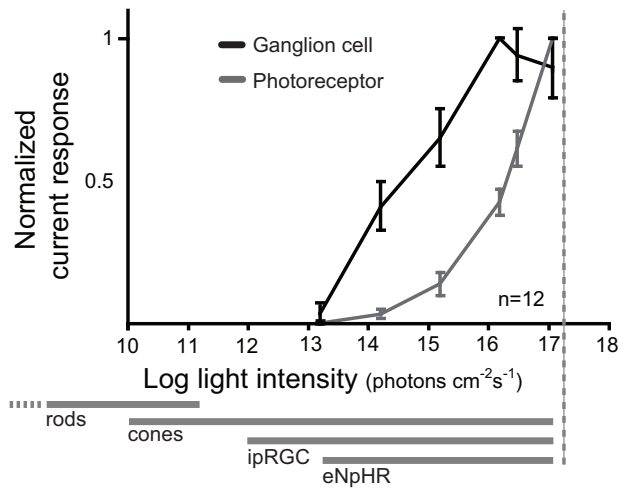


Figure 5
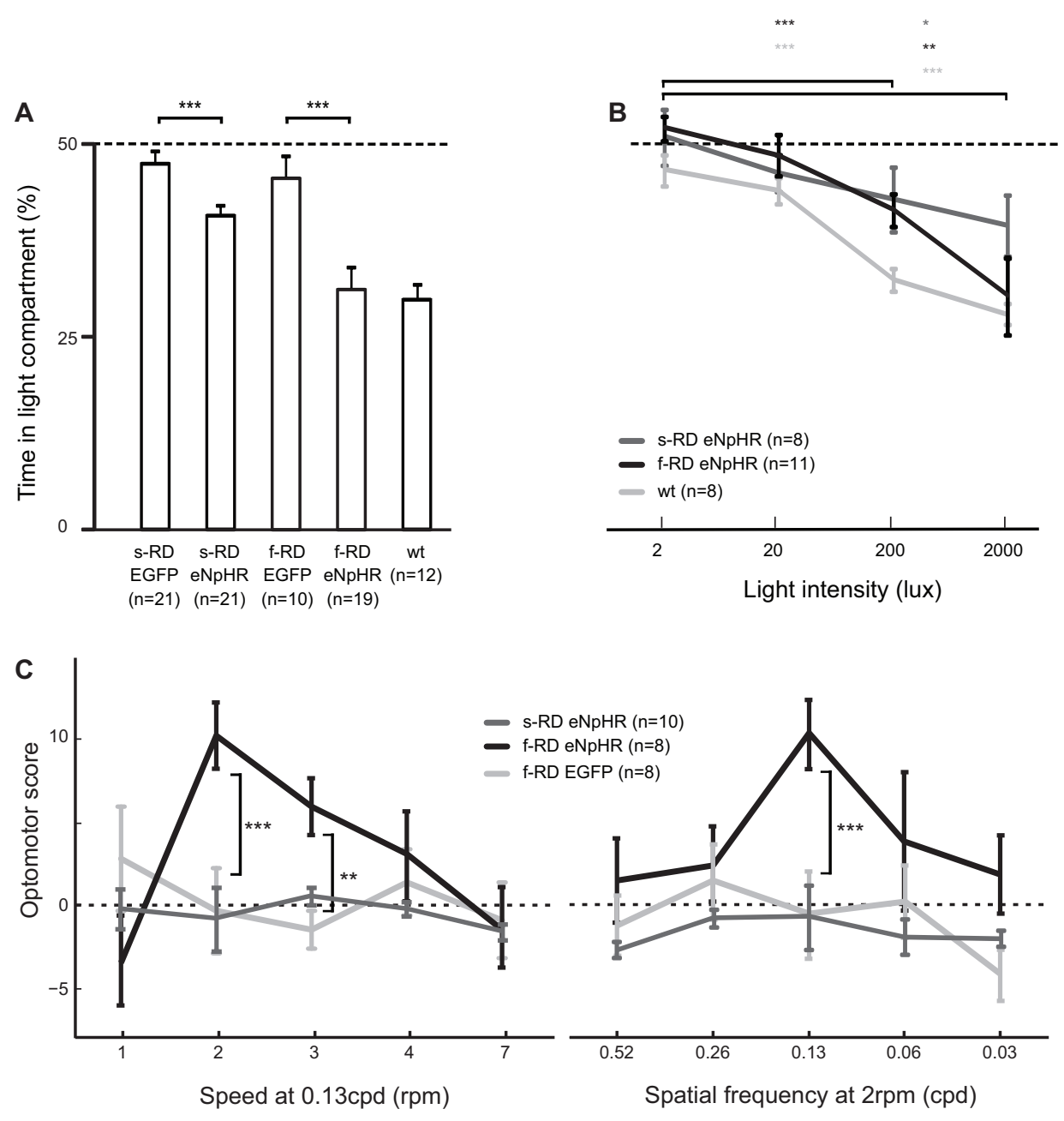
Figure 6
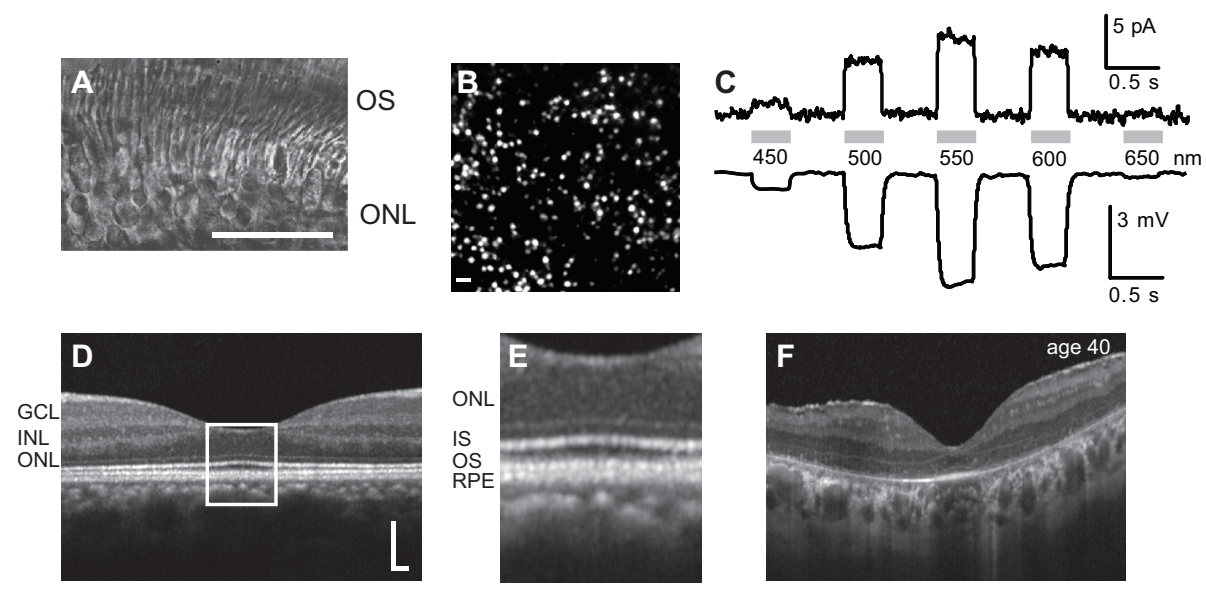